\title{
Inaudible or Unclear Audible Prompt/Feedback
}

National Cancer Institute

\section{Source}

National Cancer Institute. Inaudible or Unclear Audible Prompt/Feedback. NCI Thesaurus.

Code C63092.

Problem associated with audible prompts which cannot be heard clearly. 